\title{
Article \\ Homology-Based Interactions between Small RNAs and Their Targets Control Dominance Hierarchy of Male Determinant Alleles of Self-Incompatibility in Arabidopsis lyrata
}

\author{
Shinsuke Yasuda ${ }^{1,+, \ddagger}$, Risa Kobayashi ${ }^{1, \ddagger}$, Toshiro Ito $^{1}\left(\mathbb{D}\right.$, Yuko Wada ${ }^{1, *}$ and Seiji Takayama ${ }^{2, *}$ \\ 1 Division of Biological Sciences, Nara Institute of Science and Technology, Nara 630-0192, Japan; \\ shinsuke-yasuda@takii.co.jp (S.Y.); kobayashi.risa.ki7@bs.naist.jp (R.K.); itot@bs.naist.jp (T.I.) \\ 2 Department of Applied Biological Chemistry, Graduate School of Agricultural and Life Sciences, \\ The University of Tokyo, Tokyo 113-8657, Japan \\ * Correspondence: yu-wada@gtc.naist.jp (Y.W.); a-taka@g.ecc.u-tokyo.ac.jp (S.T.) \\ + Present address: Plant Breeding and Experiment Station, Takii and Company, Limited, \\ Konan, Shiga 520-3231, Japan. \\ $\ddagger \quad$ These authors contributed equally to this work.
}

Citation: Yasuda, S.; Kobayashi, R.; Ito, T.; Wada, Y.; Takayama, S. Homology-Based Interactions between Small RNAs and Their Targets Control Dominance Hierarchy of Male Determinant Alleles of Self-Incompatibility in Arabidopsis lyrata. Int. J. Mol. Sci. 2021, 22, 6990 https://doi.org/10.3390/ ijms22136990

Academic Editors: Silvia Coimbra and Ana Marta Pereira

Received: 30 April 2021

Accepted: 25 June 2021

Published: 29 June 2021

Publisher's Note: MDPI stays neutral with regard to jurisdictional claims in published maps and institutional affiliations.

Copyright: (c) 2021 by the authors. Licensee MDPI, Basel, Switzerland. This article is an open access article distributed under the terms and conditions of the Creative Commons Attribution (CC BY) license (https:// creativecommons.org/licenses/by/ $4.0 /)$.
Abstract: Self-incompatibility (SI) is conserved among members of the Brassicaceae plant family. This trait is controlled epigenetically by the dominance hierarchy of the male determinant alleles. We previously demonstrated that a single small RNA (sRNA) gene is sufficient to control the linear dominance hierarchy in Brassica rapa and proposed a model in which a homology-based interaction between sRNAs and target sites controls the complicated dominance hierarchy of male SI determinants. In Arabidopsis halleri, male dominance hierarchy is reported to have arisen from multiple networks of sRNA target gains and losses. Despite these findings, it remains unknown whether the molecular mechanism underlying the dominance hierarchy is conserved among Brassicaceae. Here, we identified sRNAs and their target sites that can explain the linear dominance hierarchy of Arabidopsis lyrata, a species closely related to A. halleri. We tested the model that we established in Brassica to explain the linear dominance hierarchy in A. lyrata. Our results suggest that the dominance hierarchy of $A$. lyrata is also controlled by a homology-based interaction between sRNAs and their targets.

Keywords: dominance hierarchy; self-incompatibility; epigenetics; small RNA; Brassicaceae; Arabidopsis lyrata

\section{Introduction}

Almost half of all angiosperms have a self-incompatibility (SI) system to avoid selffertilization which helps maintain genetic diversity within the species. Most Brassicaceae plants, including Arabidopsis lyrata, have SI systems ensuring that the plants' sporophytic stigmas reject their own pollen grains. This SI reaction is genetically controlled by a single multiallelic locus called the $S$ locus, which contains the pollen and stigma determinant genes S-LOCUS PROTEIN 11 (SP11, also called SCR) [1-3] and S-LOCUS RECEPTOR KINASE (SRK) [4,5], respectively. The SI reaction in Brassica rapa is caused by the $S$ haplotypespecific direct interaction between SP11 and SRK in the stigma epidermis [6,7]. These two genes are conserved in members of the Brassicaceae family, such as Brassica oleracea [4], A. lyrata [8], and Arabidopsis halleri [9].

B. rapa has a complicated dominance hierarchy among SP11 alleles, which are expressed sporophytically in anther tapetum cells. Based on analysis of pollen phenotypes, class I $\left(S_{8}, S_{9}, S_{52}, S_{12}\right) S P 11$ alleles are dominant over class-II alleles $\left(S_{44}, S_{60}, S_{40}, S_{29}\right)$ [10]. In addition, class-II alleles follow a linear dominance hierarchy: $S_{44}>S_{60}>S_{40}>S_{29}$ [10]. This complex hierarchy is controlled by interactions between just two sRNAs, SP11 methylation inducer (Smi) and Smi2, and their targets [11,12]. These sRNAs derive from inverted 
repeat sequences and silence the relatively recessive $S P 11$ allele by inducing de novo DNA methylation of its promoter region [12,13]. Smi determines the dominance-recessivity relationships between class-I and class-II SP11 alleles, and Smi2 determines the linear relationships among the four class-II SP11 alleles in a fashion dependent on the nucleotide sequences of the alleles. In particular, Smi2 and its targets contain polymorphisms to control these complex linear relationships. Thus, we previously proposed a model in which interactions between sRNAs and targets based on sequence similarity control the complicated linear dominance hierarchy of male SI determinants [12].

Compared with cultivated B. rapa, A. halleri, a wild Brassicaceae species closely related to Arabidopsis thaliana, exhibits a more divergent hierarchical pollen dominance: $\left(S_{20}, S_{13}\right)>S_{12}>S_{4}>S_{3}>S_{1}$ [14]. A model describing how this male dominance hierarchy arose from multiple networks of sRNA target gains and losses has been proposed [9]. In addition, whether dominant $S$ alleles carry a larger set of sRNAs, or whether a larger set of sRNA targets are carried by recessive $S$ alleles, has been investigated [9].

A. lyrata also exhibits a complex pollen side dominance hierarchy among $S$ haplotypes: class A2 $\left(S_{39}, S_{20}, S_{50}\right)>$ class A3 $\left(S_{13}, S_{16}\right)>$ class B $\left(S_{18}, S_{14}\right)>$ class A1 $\left(S_{1}\right)$ [14-18]. However, the mechanism underlying this dominance hierarchy, including whether it involves sRNA(s), was unknown. Here, we identified sRNAs controlling the linear dominance hierarchy in A. lyrata, and we propose a homology-dependent model to explain the dominance-recessivity interaction of SP11 in A. lyrata.

\section{Results}

\subsection{Analysis of the Dominance-Recessivity Hierarchy of the SP11 Alleles}

A. lyrata exhibits a male side dominance hierarchy of $S$ haplotypes, comprising class A2 $\left(S_{39}, S_{20}, S_{50}\right)>$ class A3 $\left(S_{13}, S_{16}\right)>$ class B $\left(S_{18}, S_{14}\right)>$ class A1 $\left(S_{1}\right)$ [14-18]. Here, for simplicity, we renamed these classes as follows: class IV $\left(S_{20}, S_{39}, S_{50}\right)>$ class III $\left(S_{13}, S_{16}\right)$ $>$ class II $\left(S_{18}, S_{14}\right)>$ class I $\left(S_{1}\right)$. We focused on the dominance-recessivity relationship between $S_{20}$-SP11 (class IV) and $S_{13}$-SP11 (class III) $\left(S_{20}>S_{13}\right)$ because the only previously reported data about such relationships in $A$. lyrata pollen was that the expression of $S_{13}-S P 11$ is reduced in a $S_{20} S_{13}$ heterozygote, as revealed by gel blot analysis and in situ hybridization [19]. First, we analyzed SP11 expression in anthers from both $S_{13} S_{13}$ homozygous and $S_{20} S_{13}$ heterozygous plants at different stages of development. Developing buds were divided into five stages based on size (Figure 1a). Reverse transcription quantitative real-time PCR (RT-qPCR) analysis showed that $S_{13}-S P 11$ transcripts started to accumulate at stage 2 (bud size: $1-2 \mathrm{~mm}$ ) and showed maximum expression at stage 4 (bud size: 3-4 mm) in $S_{13} S_{13}$ homozygotes (Figure $1 \mathrm{~b}$ ). Similarly, in a previous study, RNA gel blot analysis revealed that $S P 11$ expression in $B$. rapa anthers reaches a maximum before flower opening, when tapetum cells are intact [3]. In contrast, we found that $S_{13}$-SP11 transcript accumulation in $S_{20} S_{13}$ heterozygotes was strongly suppressed (to approximately $1 \%$ ) compared with that in $S_{13} S_{13}$ homozygotes (Figure $1 \mathrm{~b}$ ), suggesting that $S_{20}$-SP11 is dominant over $S_{13}$-SP11. This result is consistent with previously reported pollen phenotype data [14] and gel blot analysis of $S_{13}$-SP11 in $S_{20} S_{13}$ heterozygotes [8]. Therefore, we focused on the molecular mechanism of the dominance-recessivity relationship among these $S$ haplotypes.

\subsection{Identification of sRNA and Its Precursor Genes in Class-IV S Haplotypes}

To identify sRNA candidates controlling the dominance-recessivity relationships between $S_{20}$-SP11 and $S_{13}$-SP11, we performed in silico analysis of the S-locus genomic sequences of the $S_{20}$ haplotype. The aim was to identify sRNA candidate genes sharing high similarity with $S_{13}$-SP11 between the region 500 base pairs (bp) upstream from its translational initiation site and $500 \mathrm{bp}$ downstream from its termination codon. We identified an inverted repeat sequence that we named Arabidopsis lyrata SMI1 (AlSMI1) (Figure 2a), which shares high similarity with the $S_{13}$-SP11 intron region. AlSMI1 is located $27.5 \mathrm{kbp}$ downstream from $S_{20}-S P 11$ and approximately $37 \mathrm{kbp}$ from $S_{20}-S R K$. We detected 
inverted repeat sequences similar to that of $S_{20}$-AlSMI1 in both the $S_{39}$ and $S_{50}$ haplotypes (class IV) (Figure 2a).

(a)

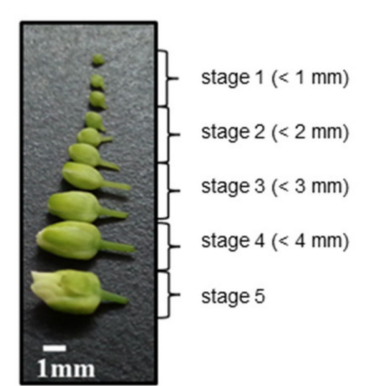

(b)

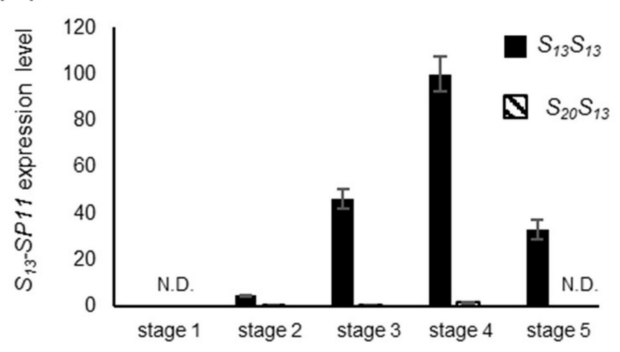

Figure 1. $S_{13}-S P 11$ expression in anthers during each stage of development and dominancerecessivity relationship between the $S_{20}$ and $S_{13}$ haplotypes. (a) Developmental stages of Arabidopsis lyrata anthers: stage $1,<1 \mathrm{~mm}$; stage $2,<2 \mathrm{~mm}$; stage $3,<3 \mathrm{~mm}$; stage $4,<4 \mathrm{~mm}$; stage 5 , the day before flowering. (b) RT-qPCR analysis of $S_{13}$-SP11 expression in $S_{13} S_{13}$ homozygotes and $S_{13} S_{20}$ heterozygotes. Elf1 $\alpha$ was used as an endogenous reference gene. The results shown are means \pm s.d. of 3 replicates. N.D., not detected.

We performed massively parallel sequencing to determine whether a 24-nucleotide (nt) sRNA is processed from $S_{20}$-AlSMI1 prior to $S_{13}-S P 11$ expression. Since $S_{13}-S P 11$ expression begins at stage 2 (Figure 1b), we analyzed sRNA from stage 1 and 2 anthers of $S_{20} S_{13}$ heterozygotes and obtained 44,553,252 sRNA sequence reads. We identified 100 reads of the 24-nt sRNA AlSmi1- $a$ (Figure 2b, Supplementary Table S1), which were processed from the $S_{20}$-AlSMI1 precursor. RNAfold [20] predicted that $S_{20}$-AlSMI1, $S_{39}$-AlSMI1, and $S_{50}-A l S M I 1$ have hairpin-structured precursors (Figure 2c). We detected AlSmi1- $a$ in the stem regions of $S_{20}$-AlSMI1, $S_{50}$-AlSMI1, and $S_{39}$-AlSMI1 (Figure 2c; Supplementary Figure S1).

We also analyzed other $S$-locus genomic sequences of the $S_{16}$ (class III), $S_{14}$ and $S_{18}$ (class II), and $S_{1}$ (class I) haplotypes, which are recessive to the $S_{20}$ haplotype in the pollen dominance hierarchy. We searched the sequences of the $S_{16^{-}}, S_{18^{-}}, S_{14^{-}}$, and $S_{1-}-S P 11$ genes $\pm 500 \mathrm{bp}$ and determined that only the AlSMI1-a region in the $S$ locus of the $S_{20}$ haplotype shares high similarity with these SP11 genes (Figure 2d; Supplementary Figures S1 and S2).

To evaluate the activity of AlSmi1- $a$, we calculated mispair scores [21] between the sRNA and $S_{13}-S P 11$. Although the mispair score system was originally developed to predict whether a 21-nt microRNA (miRNA) cuts its target, it also efficiently predicted the 24-nt Smi and Smi2 targets [12]. Mispair scores represent the number of mismatches of the complementary sites between an sRNA and its target, thus, high complementarity between an sRNA and its target leads to a lower mispair score. In our previous study, the SI phenotypes were not observed in transgenic B. rapa plants expressing Smi/Smi2 with high sequence similarity with their own SP11 (mispair scores $\leq 5.5$ ), exhibiting DNA methylation at SP11 and its silencing [11,12]. On the other hand, transgenic B. rapa plants expressing Smi2 showing low similarity with their own SP11 (mispair scores $\geq 6.5$ ), exhibited unchanged SI phenotypes [12]. Therefore, the mispair scores between dominant $S m i$ or Smi2 and the target sites of recessive SP11 were $\leq 5.5$. Here, $S_{20}$-AlSmi1- $a$ had a mispair score of $<3.0$ against the class-III SP11 intron, $<2.0$ against the class-II SP11 intron, and 4.0 against the class-I SP11 intron (Figure 2e; Supplementary Figure S2). Both $S_{39}$-AlSmi1- $a$ and $S_{50}$-AlSmi1- $a$ had mispair scores of $<5.0$ against the SP11 intron regions of all recessive $S$ haplotypes (Figure 2e; Supplementary Figure S2), suggesting that AlSmi1- $a$ potentially targets $S P 11$ in all recessive $S$ haplotypes with the same dominance relationship: class IV > (class III, class II, class I). On the other hand, although class-IV $S$ haplotypes $\left(S_{20}, S_{50}\right)$ had target sites for AlSmi1- $a$ (Figure 2d, Supplementary Figure S2), all AlSmi1- $a$ sequences had mispair scores $>7.0$ against these target sites (Figure 2e; Supplementary Figure S2). 
(a)

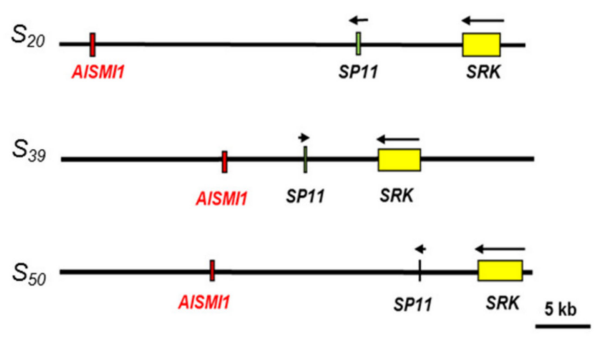

(c)

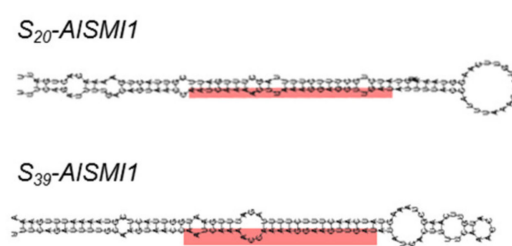

$S_{50}$-AISMI1

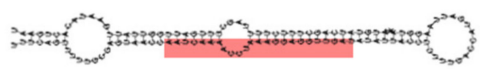

(b)

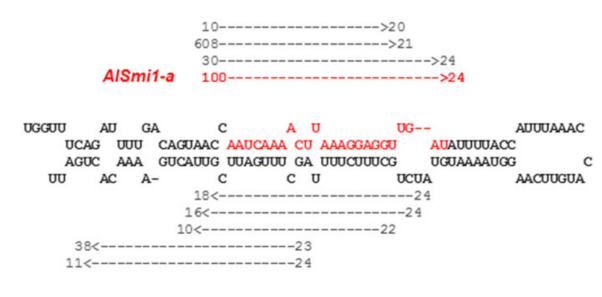

(d)

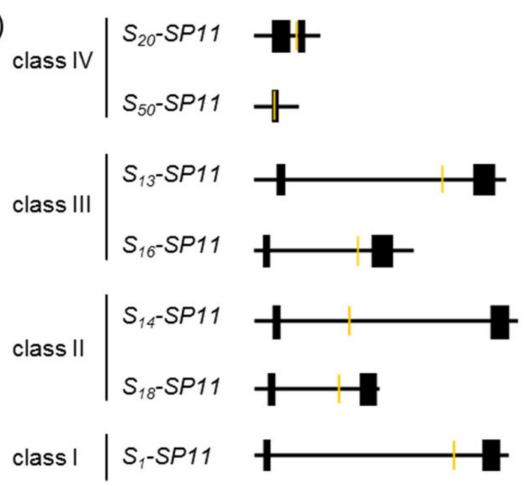

(e)

\begin{tabular}{|c|c|c|c|c|c|c|c|c|}
\hline \multirow{2}{*}{ sRNA } & \multicolumn{3}{|c|}{ class IV } & \multicolumn{2}{|c|}{ class III } & \multicolumn{2}{|c|}{ class II } & \multirow{2}{*}{$\frac{\text { class I }}{S_{1}-S P 1}$} \\
\hline & $S_{20}-S P 11$ & $S_{39}-S P 11$ & $S_{50}-S P 11$ & $S_{13}-S P 11$ & $S_{16}-S P 11$ & $S_{14}-S P 11$ & $S_{18}-S P 11$ & \\
\hline$S_{20}-A / S m i 1-a$ & 7.0 & N.D. & 18.0 & 3.0 & 3.0 & 1.0 & 2.0 & 4.0 \\
\hline$S_{39}-A / S m i 1-a$ & 11.0 & N.D. & 16.0 & 5.0 & 3.0 & 5.0 & 5.0 & 1.0 \\
\hline$S_{50}-A / S m i 1-a$ & 9.0 & N.D. & 17.0 & 5.0 & 5.0 & 3.0 & 4.0 & 3.0 \\
\hline
\end{tabular}

Figure 2. Identification of candidate sRNAs controlling the dominance-recessivity relationships: class IV > (class III, class II, class I). (a) Schematic diagrams of the $S$ locus in the $S_{20}$ haplotype (HQ379628), $S_{39}$ haplotype (KJ772418-KJ772419), and $S_{50}$ haplotype (HQ379631). Green, yellow, and red boxes indicate SP11,SRK, and AlSMI1, respectively. (b) Massively parallel sequencing analysis of sRNA expression from the AlSMI1 inverted repeat sequence. sRNAs that obtained at least 10 reads were mapped against the AISMI1 inverted repeat sequence. Arrows indicate mapped sRNAs and their directions. Numbers at the tips of arrows indicate the number of obtained sRNA reads, and numbers at the ends of arrows indicate the length of each sRNA (in nucleotides). Bases in red represent the 24-nt AlSmi1- $a$ region. (c) Stem-loop structures of AlSMI1 of the $S_{20}, S_{39}$, and $S_{50}$ haplotypes predicted by RNAfold [20]. The predicted mature 24-nt AlSmi1-a region is shown in red. (d) Schematic diagrams of the $S_{20}, S_{50}, S_{13}, S_{16}, S_{14}, S_{18}$, and $S_{1}$-SP11 genomic regions. Black boxes indicate exon regions of $S P 11$ genes, orange boxes indicate regions with high sequence similarity to AlSmi1-a. (e) Sequence similarity between AlSmi1- $a$ and SP11. Each number shows the mispair score between the class IV $\left(S_{20}, S_{39}, S_{50}\right)$-AlSmi1- $a$ and the SP11 introns of class-IV, class-III, class-II, and class-I $S$ haplotypes. N.D., not detected. Mispair scores $<5.5$ are shown in red.

Based on these results, we suggest that AlSmi1- $a$ is the only sRNA controlling the class IV > (class III, class II, class I) dominance hierarchy. On the other hand, AlSMI1 genes are also conserved in class-III and class-II $S$ locus with polymorphisms (Supplementary Figure S1).

\subsection{Identification of sRNA and Its Precursor Genes in Class-II S Haplotypes}

Next, we focused on the sequence polymorphisms of AlSMI1 among class-III and class-II $S$ haplotypes. AlSMI1 genes from the $S_{13}$ and $S_{16}$ haplotypes (class III) and the $S_{18}$ and $S_{14}$ haplotypes (class II) have similar inverted repeat structures. However, the 24-nt $S_{20}$-AlSmi1-a sequence is not conserved among either class-III or class-II $S$ haplotypes 
(Figure 3a; Supplementary Figure S1). To analyze whether AlSmi1 is processed in class-III $S$ haplotypes, we mapped reads obtained by the massively parallel sequencing of $S_{20} S_{13}$ against the $S_{13}$-AlSMI1 inverted repeat sequence. No reads were mapped onto the $S_{13^{-}}$ AlSMI1 precursor. These results suggest that $S_{13}$-AlSMI1 is not processed into 24-nt sRNAs and that AlSMI1 is not functional in this class-III $S$ haplotype (Figure 3a).

(a)

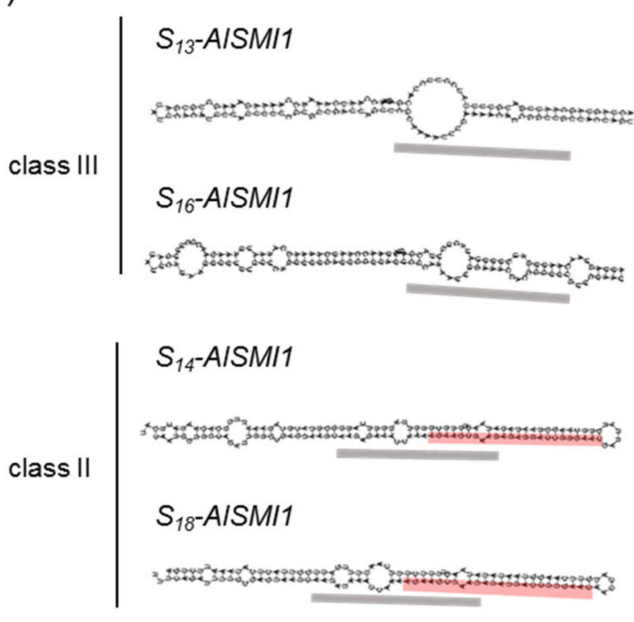

(b)

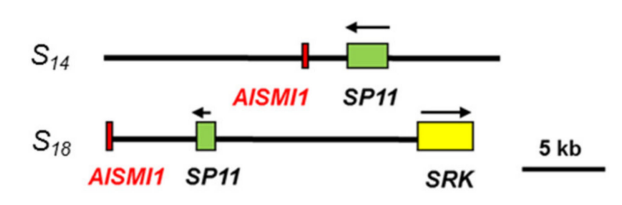

(c)

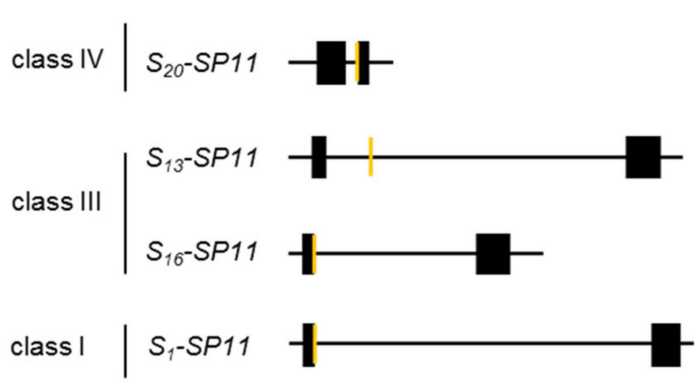

(d)

\begin{tabular}{|c|c|c|c|c|c|c|c|c|}
\hline \multirow{2}{*}{ sRNA } & \multicolumn{3}{|c|}{ class IV } & \multicolumn{2}{|c|}{ class III } & \multicolumn{2}{|c|}{ class II } & \multirow{2}{*}{$\frac{\text { class I }}{S_{1}-S P 11}$} \\
\hline & $S_{20}-S P 11$ & $S_{39}-S P 11$ & $S_{50}-S P 11$ & $S_{13}-S P 11$ & $S_{16}-S P 11$ & $S_{14}-S P 11$ & $S_{18}-S P 11$ & \\
\hline class-II-A/Smi1-b & 9.0 & N.D. & N.D. & 7.0 & 9.0 & N.D. & N.D. & 3.0 \\
\hline
\end{tabular}

Figure 3. Identification of sRNAs controlling the dominance-recessivity relationships: class II > class I. (a) Stem-loop structures of the $S_{13}, S_{16}, S_{14}$, and $S_{18}$ haplotypes of AlSMI1 predicted by RNAfold [20]. Black dashed boxes indicate 24-nt snRNAs corresponding to class-III and class-II AlSmi1-a. The predicted 24-nt mature AlSmi1-b is shown in red. (b) Schematic diagrams of the $S$ locus in the $S_{14}$ haplotype (KJ772406) and $S_{18}$ haplotype (KJ772412). Green, yellow, and red boxes indicate $S P 11, S R K$, and AlSMI1, respectively. (c) Schematic diagrams of the SP11 genomic regions of the $S_{20}, S_{13}, S_{16}$, and $S_{1}$ haplotypes. Black boxes indicate exon regions of $S P 11$, orange boxes indicate the target regions homologous to AlSmi1- $b$. (d) Sequence similarity between AlSmi1- $b$ and SP11. Numbers are the mispair scores between class-II AlSmi1- $b$ and class-IV, class-III, class-II, and class-I $S$ haplotypes of SP11. N.D., not detected. Mispair scores $<5.5$ are shown in red.

Interestingly, $S_{18}$ and $S_{14}$-AlSMI1 (class II) contain another 24-nt sequence (AlSmi1-b) that shares high similarity with a relatively recessive $S_{1}$-SP11 (class I) exon junction (mispair score 3.0) (Figure 3a-d; Supplementary Figures S1 and S2). This suggests that sRNA processed from this region might be involved in the dominance relationship between class II and class I (class II $>$ class I). AlSmi1- $b$ had a mispair score $>7.0$ against $S_{20}-S P 11$ (class IV) and $S_{13}, S_{16}-S P 11$ (class III), consistent with dominance hierarchy (class IV and class III > class II) (Figure 3c,d; Supplementary Figure S2). These results suggest that the interaction between AlSmi1- $a$ from class-IV $S$ haplotypes and its target site at the SP11 intron of recessive class-III, class-II, and class-I $S$ haplotypes controls the dominance relationships among these $S$ haplotypes. On the other hand, the interaction between AlSmi1- $b$ from class-II $S$ haplotypes and its target site at the SP11 exon junction of recessive class-I $S$ haplotypes controls the dominance relationships among these $S$ haplotypes.

\subsection{Identification of sRNA and Its Precursor Genes in Class-III $S$ Haplotypes}

Since processed $S_{13}$-AlSmi1 was not detected in $S_{20} S_{13}$ heterozygotes, we searched for other sRNAs that could control the dominance relationship class III $>$ (class II and class I). We searched the $S$ locus genomic sequence of the $S_{13}$ haplotype, which shares high 
similarity with class-II $S_{14}$ - and $S_{18}$-SP11 and class-I $S_{1}$-SP11 genes $\pm 500 \mathrm{bp}$. We identified another inverted repeat sequence with high similarity to the 30 -bp region upstream from the translation initiation sites of class-I and class-II SP11 genes, which we named A. lyrata SMI2 (AlSMI2) (Figure 4a). A database search identified the AlSMI2 sequence only in the class-III $S_{13}$ and $S_{16}$ haplotypes (Figure $4 a$ ).

(a)

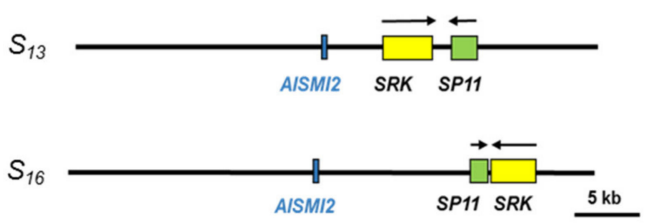

(c)

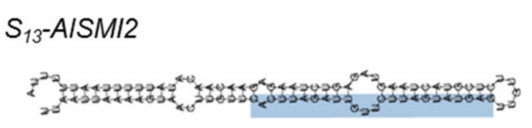

$S_{16}-A / S M I 2$

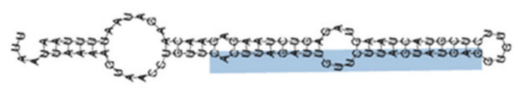

(b)

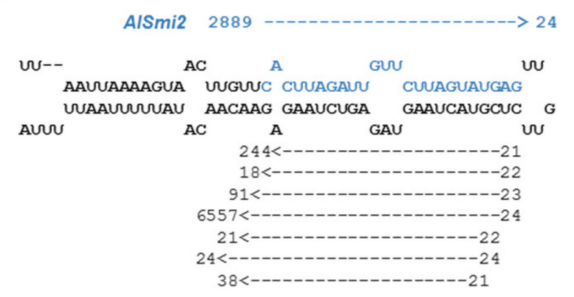

(d)

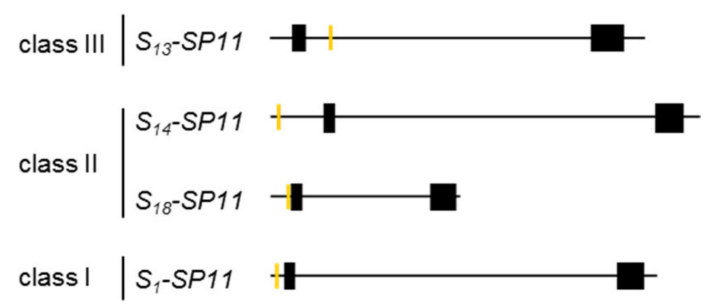

(e)

\begin{tabular}{|c|c|c|c|c|c|c|c|c|}
\hline & \multicolumn{3}{|c|}{ class IV } & \multicolumn{2}{|c|}{ class III } & \multicolumn{2}{|c|}{ class II } & \multirow{2}{*}{$\frac{\text { class I }}{S_{1}-S P 11}$} \\
\hline sRNA & $S_{20}-S P 11$ & $S_{39}-S P 11$ & $S_{50}-S P 11$ & $S_{13}-S P 11$ & $S_{16}-S P 11$ & $S_{14}-S P 11$ & $S_{18}-S P 11$ & \\
\hline AlSmi2 & N.D. & N.D. & N.D. & 6.5 & N.D. & 4.5 & 4.5 & 3.5 \\
\hline
\end{tabular}

Figure 4. Identification of the candidate sRNAs controlling dominance-recessivity relationships: class III > (class II, class I). (a) Schematic diagrams of the $S$ locus in the $S_{13}$ haplotype (ADBK01001387) and the $S_{16}$ haplotype (HQ379629). Green, yellow, and blue boxes indicate SP11, SRK, and AlSMI2, respectively. (b) Massively parallel sequencing analysis of sRNA expression from the $S_{13}$-AlSMI2 inverted repeat sequence. sRNAs obtained from more than 10 reads were mapped against the $S_{13}$-AlSMI2 inverted repeat sequence. Arrows indicate mapped sRNAs and their directions. Numbers at the tips of arrows indicate the obtained read numbers, and numbers at the ends of arrows indicate the base length of each sRNA. Bases in blue indicate the 24-nt AlSmi2 region. (c) Stem-loop structures of AlSMI2 of the $S_{13}$ and $S_{16}$ haplotypes predicted by RNAfold [20]. The predicted 24-nt mature AlSmi2 is shown in blue. (d) Schematic diagrams of the SP11 genomic regions of the $S_{13}, S_{14}, S_{18}$, and $S_{1}$ haplotypes. Black boxes indicate exon regions of $S P 11$, orange boxes indicate the target region homologous to AlSmi2. (e) Sequence similarity between AlSmi2 and SP11. Each number indicates the mispair score between class-III AlSmi2 and class-IV, class-III, class-II, and class-I S haplotypes of SP11. N.D., not detected. Mispair scores < 5.5 are shown in red.

We mapped sRNA sequence reads derived from the anthers of $S_{20} S_{13}$ heterozygotes against the $S_{13}$-AlSMI2 region and identified 2,889 AlSmi2 reads sharing high similarity with the $5^{\prime}$ upstream region of SP11 (Figure 4b; Supplementary Table S1). RNAfold [20] predicted that $S_{13}$-AlSMI2 and $S_{16}$-AlSMI2 had hairpin-structured precursors (Figure 4c). The mature 24-nt AlSmi2 sequence was identified in the stem regions of $S_{13}$-AlSMI2 and $S_{16}$-AlSMI2 (Figure 4c).

AlSmi2 shares high similarity with the $5^{\prime}$ regions of the relatively recessive haplotypes $S_{14^{-}}$and $S_{18^{-}}$SP11 (class II) and $S_{1}-S P 11$ (class I), with mispair scores $<4.5$ (Figure 4 d,e; Supplementary Figure S2). Although $S_{13}-S P 11$ intron contains a candidate target site of AlSmi2, it showed low similarity with this sequence (mispair score 6.5) (Figure 4d,e; Supplementary Figure S2). These results suggest that AlSmi2 controls the dominance 
relationship class III > (class II, class I) as a result of its high similarity (mispair score $<4.5$ ) with the SP11 sequences of relatively recessive $S$ haplotypes.

\subsection{Quantification of a 24-nt sRNA and Its Precursor}

To confirm the expression of $S_{20}$-AlSMI1 and $S_{13}$-AlSMI2, which are precursors of $S_{20}$-AlSmi1- $a$ and $S_{13}$-AlSmi2, respectively, in $S_{20} S_{13}$ heterozygotes, we performed RT-qPCR (Figure 5a). In B. rapa, the expression of Smi1 and Smi2 is induced before the initiation of SP11 transcription [13]. Since SP11 transcription is initiated at stage 2 in A. lyrata (Figure 1b), we analyzed $S_{20}$-AlSMI1 and $S_{13}$-AlSMI2 precursors in stage 1 and 2 anthers. We detected strong accumulation of both the $S_{20}$-AlSMI1 and $S_{13}$-AlSMI2 precursors at stage 1 , which then declined by stage 2 (Figure $5 \mathrm{a}$ ). This expression pattern suggests that $S_{20}$-AlSMI1 and $S_{13}-A I S M I 2$ might act to suppress SP11. Additionally, we analyzed the expression of mature 24-nt $S_{20}$-AlSmi1- $a$ and $S_{13}$-AlSmi2 in early stage anthers (stages 1-2) of $S_{20} S_{13}$ heterozygotes via stem-loop RT-qPCR of RNA samples [22] (Figure 5b). The results suggest that the precursors of these sRNAs and mature 24-nt sRNAs are expressed in vivo.

(a)

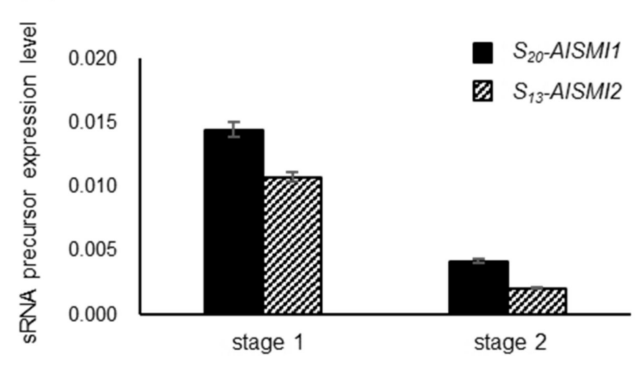

(b)

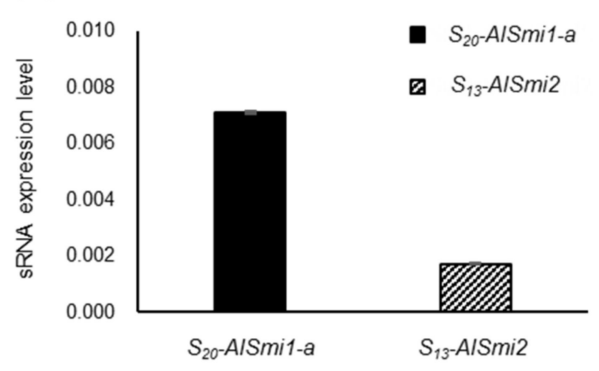

Figure 5. Accumulation of sRNA precursors and a 24-nt sRNA in early stage anthers of $S_{20} S_{13}$ heterozygotes. (a) RT-qPCR of $S_{13}$-AlSMI2 and $S_{20}$-AlSMI1 precursors at stages 1 and 2 in $S_{20} S_{13}$ heterozygotes. GAPDH was used as an endogenous reference gene. The results shown are means \pm s.d. of 3 replicates. (b) Quantification of $S_{13}-A l S m i 2$ and $S_{20}-A l S m i 1$ in mixtures of stage 1 and 2 anthers in $S_{20} S_{13}$ heterozygotes via stem-loop RT-PCR analysis. miR166 was used as an endogenous reference gene. The results shown are means \pm s.d. of 3 replicates.

\section{Discussion}

In this study, we identified two sRNAs homologous to SP11 genes in A. lyrata. The accumulation of the $S_{13}-S P 11$ transcript in $S_{20} S_{13}$ heterozygotes was strongly suppressed (to approximately $1 \%$ ) compared with that in $S_{13} S_{13}$ homozygotes (Figure 1b), suggesting that class-IV $S_{20}-S P 11$ is dominant over class-III $S_{13}-S P 11$. AlSmi1-a from class IV is thought to be the only sRNA that controls the relationship class IV > (class III, class II, class I) (Figure 2a-e). Class-II and class-III AlSMI1 gene sequences with polymorphisms are also conserved (Supplementary Figure S1). Class II-specific AlSmi1-b shares high similarity with a relatively recessive $S_{1}-S P 11$ (class I) exon junction (mispair score 3.0) (Figure 3a-d; Supplementary Figure S1 and S2), suggesting that sRNA processed from this region might be involved in the dominance relationship between class-II and class-I sRNAs (class II > class I). Another sRNA, AlSmi2, which shares high similarity with SP11 genes of relatively recessive $S$ haplotypes, is involved in the dominance relationship class III $>$ (class II, class I) (Figure $4 \mathrm{a}-\mathrm{e}$ ). The accumulation of both $S_{20}$-AlSMI1 and $S_{13}$-AlSMI2 precursors was detected (Figure 5a), and mature 24-nt sRNAs of $S_{20}$-AlSmi1- $a$ and $S_{13}$-AlSmi2 were expressed in vivo (Figure $5 b$ ). These results suggest that the linear dominance hierarchy in $A$. lyrata is controlled by two polymorphic sRNAs, AlSmi1 and AlSmi2 (Figure 6). 


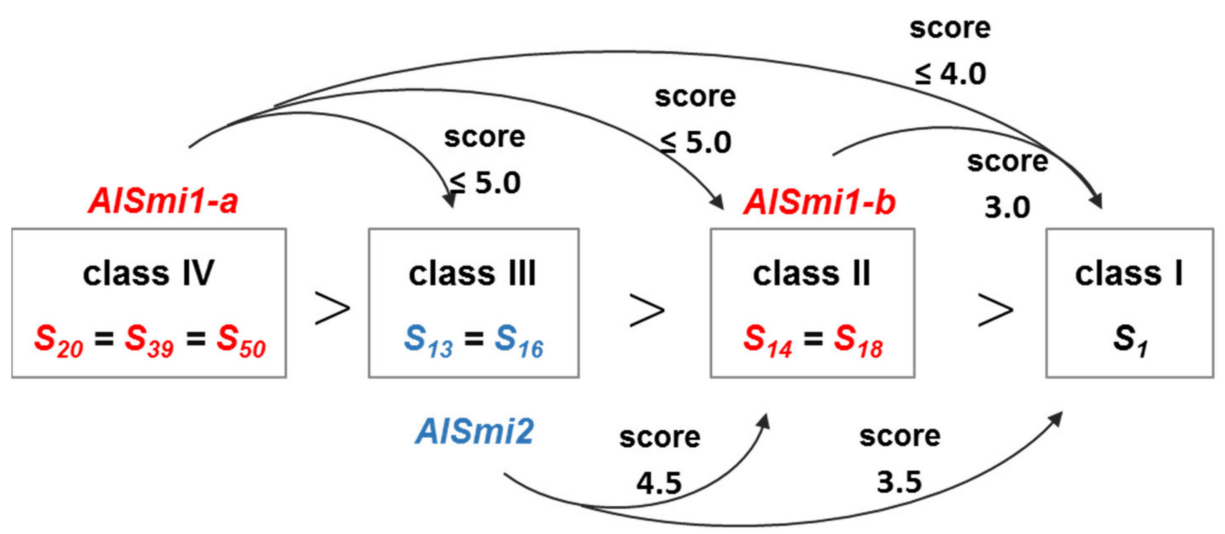

Figure 6. Regulatory network of the identified sRNAs and their predicted targets in A. lyrata. ClassIV $S_{20}$-SP11 is dominant over class-III $S_{13}$-SP11 (Figure 1). AlSmi1- $a$ from class-IV $S$ haplotypes is homologous to SP11 from recessive $S$ haplotypes (classes III, II, and I), showing a mispair score $<5.0$ (Figure 2). AlSmi1- $b$ predicted from class-II $S$ haplotypes is homologous to a class-I SP11, showing a mispair score $<3.0$ (Figure 3). AlSmi2 from class-III $S$ haplotypes is homologous to SP11 from relatively recessive $S$ haplotypes (classes II and I), showing a mispair score $<4.5$ (Figure 4 ). Both precursors and mature 24-nt sRNA of AlSmi1- $a$ and AlSmi2 were detected in vivo (Figure 5).

AlSmi1 and AlSmi2 correspond to mirS3 and mir1887 in A. halleri, respectively [9]. A species-wide survey of sequence diversity revealed that a large fraction of alleles at the pistil side SI determinant SRK are trans-specifically shared between A. lyrata and A. halleri [23]. Our findings suggest that not only $S R K$ but also other $S$-locus genes, including sRNAs, are shared between $A$. lyrata and $A$. halleri. These sRNAs are conserved in both species in the regulation of pollen side dominance. A pollen side linear dominance hierarchy controlled by sRNAs has also been identified in B. rapa [11,12], pointing to a common sRNA-based dominance-recessivity mechanism in Brassicaceae plants.

Furthermore, our data suggest that the dominance-recessivity mechanism in both B. rapa and A. lyrata is explained by the sequence homology between sRNA and its target. In our previous report, the sRNA from the dominant allele had a mispair score $<5.5$ against the target region of recessive SP11. In the current study, AlSmi1 and AlSmi2 in A. lyrata also had mispair scores $<5.0$ against relatively recessive $S P 11$ s (Figures 2e, $3 \mathrm{~d}$, 4e and 6; Supplementary Figure S2). Concurrently, these sRNAs had a mispair score $>6.5$ against self- and relatively dominant SP11s (Figures 2e, 3d and 4e; Supplementary Figure S2). Two models have been examined in A. halleri: (1) dominant $S$ alleles carry a larger set of sRNAs; and (2) recessive $S$ alleles carry a larger set of sRNA targets [9]. Here, we identified only one sRNA, AlSmi1- $a$, from the most dominant $S$ haplotype (class IV), and found that recessive $S$ haplotypes did not contain a larger number of sRNA targets (Figures $2 \mathrm{~b}, 3 \mathrm{c}$ and $4 \mathrm{~d}$ ). These results strongly suggest that the polymorphic dominance modifier model we proposed for $B$. rapa also explains the dominance relationships in A. lyrata. Further analysis of the role of the interactions between sRNAs and their target regions should provide support for this model.

Similar complex dominance-recessivity relationships can be observed in other SI plants of the Asteraceae and Convolvulaceae [24,25]. Moreover, dominance relationships have been reported among mimicry genes in butterflies [26] and among genes in various plants and animals [27-30]. Further studies are needed to determine whether our model can explain these complicated dominance-recessivity networks and monoallelic gene expression. 


\section{Materials and Methods}

\subsection{Plant Materials}

A. lyrata SaSb seeds were a gift from Y. Takada (Tohoku University). For all experiments, floral buds from clonally propagated progenies of a single individual were used. The

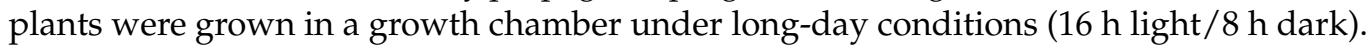

\section{2. $R T-q P C R$ of $S P 11$}

Total RNA was isolated from the anthers of $S_{13} S_{13}$ homozygotes and $S_{20} S_{13}$ heterozygotes at each stage of development and used for RT-qPCR, as previously described [13]. The $S_{13}$-SP11 region was amplified using specific primers: forward primer, 5'-AGCCATGTTCAA GGAATGGAAGA-3'; reverse primer, 5'-TTGTTGCCATCCTCCGTAAGGTC-3'. Elf1 $\alpha$ was used as an endogenous reference gene and amplified using specific primers: forward primer, 5'-TGGTGACGCTGGTATGGTTA-3'; reverse primer, 5'-GGTCTGCCTCATGTCCC TAA-3'.

\subsection{Prediction of Inverted Repeat Sequence Regions}

Inverted repeat sequences were searched from the $S$-locus sequence data of each haplotype: $S_{20}$ (HQ379628), $S_{39}$ (KJ772415-KJ772419), $S_{50}$ (HQ379631), $S_{13}$ (ADBK01001387), $S_{16}$ (HQ379629), $S_{14}$ (KJ772405-KJ772407), $S_{18}$ (KJ772408-KJ772414), and $S_{1}$ (KJ772401KJ772404). The $S$ locus was defined as the region between PUB8 (At4g21350) and ARK3 (At4g21380), as described previously [15]. First, each SP11 gene containing both upstream and downstream 500-bp fragments was divided into 500-bp fragments overlapping every 30 -bp region. These fragments were used as queries in BLAST searches against the $S$ loci of the $S_{20}$ and $S_{13}$ haplotypes. Sequences showing $E$-values $<1 \times 10^{-3}$ were further analyzed. The secondary structures of the precursors from the obtained inverted repeat were predicted using RNAfold [20]. Hairpin-structured miRNA-like sequences having low energy values under $-30 \mathrm{kcal} \mathrm{mol}^{-1}$ were screened.

\subsection{Small RNA Sequencing and Mapping}

Total RNA containing the sRNA fraction was isolated from a mixture of stage 1 and 2 anthers using a mirVana miRNA Isolation Kit (Ambion/Thermo Fisher Scientific, Waltham, MA, USA). Preparation of the small RNA library, Illumina GAII sequencing, and adaptor trimming of sequencing reads were performed at Hokkaido System Science Co., Ltd (Sapporo, Japan). sRNA sequences with more than 10 reads were mapped against the $S$ locus of the $S_{20}$ haplotype (HQ379628) and the $S_{13}$ haplotype (ADBK01001387). We also mapped the obtained reads against the predicted precursors of AlSMI1 and AlSMI2 using the Bowtie program [31]. The mispair score was calculated as described [21]: mismatches were scored as 1, and G:U pairs were scored as 0.5 . Mismatched and G:U pair scores within the core segment were doubled.

\subsection{RT-qPCR of sRNA Precursor}

Total RNA was isolated from anthers of $S_{20} S_{13}$ heterozygotes at each stage of development using an RNeasy Mini Kit (QIAGEN GmbH, Hilden, Germany) and used for RT-qPCR, as previously described [13]. Each sRNA precursor region was amplified using specific primers: $S_{20}$-AlSMI1 forward primer, 5'-AGCAATGGTTTCAGATTTTGACAGTAACC-3'; $S_{20}$-AlSMI1 reverse primer, 5'-AGATACATTTTACCTTGAACATGGTTTAAATGG-3'; $S_{13^{-}}$ AlSMI2 forward primer, 5'-TTAATTAAAAGTAACTTGTTCACTTAGATTGTTCTTAG-3'; $S_{13}$-AlSMI2 reverse primer, 5'-ATGTTGTTCTCTTAGACTCTACTTAGTACG-3'. GAPDH was used as an endogenous reference gene and amplified using specific primers: forward primer, 5'-GACCTTACTGTCAGACTCGAG-3'; reverse primer, 5'-CGGTGTATCCAAGGAT TCCCT-3'. 


\subsection{Stem-Loop RT-PCR}

Quantification of 24-nt $S_{20}$-AlSmi1 and $S_{13}$-AlSmi2 sRNAs was performed as previously described [22]. sRNAs were isolated from anthers (mixture of stages 1 and 2) from $S_{20} S_{13}$ heterozygotes using a mirVana miRNA Isolation Kit (Ambion/Thermo Fisher Scientific, Waltham, MA, USA) and reverse transcribed using a TaqMan MicroRNA Reverse Transcription Kit (Applied Biosystems/Thermo Fisher Scientific, Waltham, MA, USA) with 24-nt sRNA-specific RT primers: $S_{20}$-AlSmi1 RT-forward primer, 5'-GTTGGCTCTGGTGCAGGGT CCGAGGTATTCGCACCAGA-3'; $S_{13}$-AlSmi2 RT-forward primer, 5'-GTTGGCTCTGGTGC AGGGTCCGAGGTATTCGCACCAGA-3'. The reverse transcription products were quantified using Light Cycler 480 Probes Master Mix (Roche, Basel, Switzerland) and Universal Probe Library \#21 (Roche) with small RNA-specific primers and universal primers: $S_{20}$-AlSmi1 forward primer, 5'-CGGCGGCAATCAAAACTTAAAGGAG-3'; $S_{13}$-AlSmi2 forward primer, 5'-CGGCGGCCACTTAGATTGTTCTTAG-3'; universal primer, 5'-GTGCAGG GTCCGAGGT-3'. miR166 was used as an endogenous reference miRNA and was amplified using specific primers: RT-forward primer, $5^{\prime}$-GTTGGCTCTGGTGCAGGGTCCGAGGTAT TCGCACCAGAGCCAACGGGGAA-3'; forward primer, 5'-CAGCATCGGACCAGGCTT CA-3'.

Supplementary Materials: Supplementary materials can be found at https://www.mdpi.com/ article/10.3390/ijms22136990/s1.

Author Contributions: S.Y., Y.W. and S.T. designed the research; S.Y. and R.K. performed the research; S.Y., R.K. and Y.W. analyzed data; R.K., T.I., Y.W. and S.T. wrote the article. All authors have read and agreed to the published version of the manuscript.

Funding: This work was supported in part by Grants-in-Aid for Scientific Research (16H06380 to S.T. and Y.W.; $20 \mathrm{H00470}$ to T.I. and Y.W.; 19H04865 to T.I.; 19K06781 and 18H02456 to Y.W.; $16 \mathrm{H} 06467$ to S.T.), a Grant-in-Aid for JSPS Research Fellow (14J10324 to S.Y.), and a grant from the Takeda Science Foundation to Y.W.

Data Availability Statement: Sequence data for $S_{20}-A I S M I 1$ and $S_{13}$-AlSMI2 have been deposited in DDBJ under accession numbers LC629453 and LC629454.

Acknowledgments: We thank Y. Takada for helpful comments and F. Kodama, M. Nara, and Y. Yoshimura for technical assistance.

Conflicts of Interest: The authors declare that the research was conducted in the absence of any commercial or financial relationships that could be construed as a potential conflict of interest.

\section{References}

1. Suzuki, G.; Kai, N.; Hirose, T.; Fukui, K.; Nishio, T.; Takayama, S.; Isogai, A.; Watanabe, M.; Hinata, K. Genomic organization of the $S$ locus: Identification and characterization of genes in SLG/SRK region of $S^{9}$ haplotype of Brassica campestris (syn. rapa). Genetics 1999, 153, 391-400. [CrossRef]

2. Schopfer, C.R.; Nasrallah, M.E.; Nasrallah, J.B. The male determinant of self-incompatibility in Brassica. Science 1999, 286, 1697-1700. [CrossRef]

3. Takayama, S.; Shiba, H.; Iwano, M.; Shimosato, H.; Che, F.S.; Kai, N.; Watanabe, M.; Suzuki, G.; Hinata, K.; Isogai, A. The pollen determinant of self-incompatibility in Brassica campestris. Proc. Natl. Acad. Sci. USA 2000, 97, 1920-1925. [CrossRef]

4. Stein, J.C.; Howlett, B.; Boyes, D.C.; Nasrallah, M.E.; Nasrallah, J.B. Molecular cloning of a putative receptor protein kinase gene encoded at the self-incompatibility locus of Brassica oleracea. Proc. Natl. Acad. Sci. USA 1991, 88, 8816-8820. [CrossRef]

5. Takasaki, T.; Hatakeyama, K.; Suzuki, G.; Watanabe, M.; Isogai, A.; Hinata, K. The $S$ receptor kinase determines self-incompatibility in Brassica stigma. Nature 2000, 403, 913-916. [CrossRef]

6. Kachroo, A.; Schopfer, C.R.; Nasrallah, M.E.; Nasrallah, J.B. Allele-specific receptor-ligand interactions in Brassica selfincompatibility. Science 2001, 293, 1824-1826. [CrossRef]

7. Takayama, S.; Shimosato, H.; Shiba, H.; Funato, M.; Che, F.S.; Watanabe, M.; Iwano, M.; Isogai, A. Direct ligand-receptor complex interaction controls Brassica self-incompatibility. Nature 2001, 413, 534-538. [CrossRef]

8. Kusaba, M.; Dwyer, K.; Hendershot, J.; Vrebalov, J.; Nasrallah, J.B.; Nasrallah, M.E. Self-incompatibility in the genus Arabidopsis: Characterization of the $S$ locus in the outcrossing A. lyrata and its autogamous relative A. thaliana. Plant Cell. 2001, 13, 627-644. [CrossRef] 
9. Durand, E.; Méheust, R.; Soucaze, M.; Goubet, P.M.; Gallina, S.; Poux, C.; Fobis-Loisy, I.; Guillon, E.; Gaude, T.; Sarazin, A.; et al. Dominance hierarchy arising from the evolution of a complex small RNA regulatory network. Science 2014, 346, $1200-1205$. [CrossRef]

10. Hatakeyama, K.; Watanabe, M.; Takasaki, T.; Ojima, K.; Hinata, K. Dominance relationships between S-alleles in self-incompatible Brassica campestris L. Heredity 1998, 80, 241-247. [CrossRef]

11. Tarutani, Y.; Shiba, H.; Iwano, M.; Kakizaki, T.; Suzuki, G.; Watanabe, M.; Isogai, A.; Takayama, S. Trans-acting small RNA determines dominance relationships in Brassica self-incompatibility. Nature 2010, 466, 983-986. [CrossRef] [PubMed]

12. Yasuda, S.; Wada, Y.; Kakizaki, T.; Tarutani, Y.; Miura-Uno, E.; Murase, K.; Fujii, S.; Hioki, T.; Shimoda, T.; Takada, Y.; et al. A complex dominance hierarchy is controlled by polymorphism of small RNAs and their targets. Nat. Plants 2016, $3,16206$. [CrossRef]

13. Shiba, H.; Kakizaki, T.; Iwano, M.; Tarutani, Y.; Watanabe, M.; Isogai, A.; Takayama, S. Dominance relationships between self-incompatibility alleles controlled by DNA methylation. Nat. Genet. 2006, 38, 297-299. [CrossRef]

14. Prigoda, N.L.; Nassuth, A.; Mable, B.K. Phenotypic and genotypic expression of self-incompatibility haplotypes in Arabidopsis lyrata suggests unique origin of alleles in different dominance classes. Mol. Biol. Evol. 2005, 22, 1609-1620. [CrossRef] [PubMed]

15. Guo, Y.L.; Zhao, X.; Lanz, C.; Weigel, D. Evolution of the S-locus region in Arabidopsis relatives. Plant Physiol. 2011, 157, 937-946. [CrossRef] [PubMed]

16. Goubet, P.M.; Bergès, H.; Bellec, A.; Prat, E.; Helmstetter, N.; Mangenot, S.; Gallina, S.; Holl, A.C.; Fobis-Loisy, I.; Vekemans, X.; et al. Contrasted patterns of molecular evolution in dominant and recessive self-incompatibility haplotypes in Arabidopsis. PLoS Genet. 2012, 8, e1002495. [CrossRef] [PubMed]

17. Schierup, M.H.; Mable, B.K.; Awadalla, P.; Charlesworth, D. Identification and characterization of a polymorphic receptor kinase gene linked to the self-incompatibility locus of Arabidopsis lyrata. Genetics 2001, 158, 387-399. [CrossRef]

18. Mable, B.K.; Beland, J.; Di Berardo, C. Inheritance and dominance of self-incompatibility alleles in polyploid Arabidopsis lyrata. Heredity 2004, 93, 476-486. [CrossRef]

19. Kusaba, M.; Tung, C.W.; Nasrallah, M.E.; Nasrallah, J.B. Monoallelic expression and dominance interactions in anthers of self-incompatible Arabidopsis lyrata. Plant Physiol. 2002, 128, 17-20. [CrossRef]

20. Lorenz, R.; Bernhart, S.H.; Höner zu Siederdissen, C.; Tafer, H.; Flamm, C.; Stadler, P.F.; Hofacker, I.L. ViennaRNA package 2.0. Algorithms Mol. Biol. 2011, 6, 26-39. [CrossRef]

21. Fahlgren, N.; Carrington, J.C. miRNA target prediction in plants. Methods Mol. Biol. 2010, 592, 51-57. [CrossRef] [PubMed]

22. Chen, C.; Ridzon, D.A.; Broomer, A.J.; Zhou, Z.; Lee, D.H.; Nguyen, J.T.; Barbisin, M.; Xu, N.L.; Mahuvakar, V.R.; Andersen, M.R.; et al. Real-time quantification of microRNAs by stem-loop RT-PCR. Nucleic Acids Res. 2005, 33, e179. [CrossRef]

23. Castric, V.; Bechsgaard, J.; Schierup, M.H.; Vekemans, X. Repeated Adaptive introgression at a gene under multiallelic balancing selection. PLoS Genet. 2008, 4, e1000168. [CrossRef]

24. Kowyama, Y.; Takahasi, H.; Muraoka, K.; Tani, T.; Hara, K.; Shiotani, I. Number, frequency and dominance relationships of S-alleles in diploid Ipomoea trifida. Heredity 1994, 73, 275-283. [CrossRef]

25. Brennan, A.C.; Tabah, D.A.; Harris, S.A.; Hiscock, S.J. Sporophytic self-incompatibility in Senecio squalidus (Asteraceae): S allele dominance interactions and modifiers of cross-compatibility and selfing rates. Heredity 2011, 106, 113-123. [CrossRef] [PubMed]

26. Joron, M.; Papa, R.; Beltrán, M.; Chamberlain, N.; Mavárez, J.; Baxter, S.; Abanto, M.; Bermingham, E.; Humphray, S.J.; Rogers, J.; et al. A conserved supergene locus controls colour pattern diversity in Heliconius butterflies. PLoS Biol. 2006, 4, e303. [CrossRef]

27. Guo, M.; Rupe, M.A.; Zinselmeier, C.; Habben, J.; Bowen, B.A.; Smith, O.S. Allelic variation of gene expression in maize hybrids. Plant Cell 2004, 16, 1707-1716. [CrossRef]

28. Gimelbrant, A.; Hutchinson, J.N.; Thompson, B.R.; Chess, A. Widespread monoallelic expression on human autosomes. Science 2007, 318, 1136-1140. [CrossRef]

29. Zhuang, Y.; Adams, K.L. Extensive allelic variation in gene expression in populus $\mathrm{F}_{1}$ hybrids. Genetics 2007, 177, 1987-1996. [CrossRef]

30. Wang, J.; Valo, Z.; Smith, D.; Singer-Sam, J. Monoallelic expression of multiple genes in the CNS. PLoS ONE 2007, 2, e1293. [CrossRef]

31. Langmead, B.; Trapnell, C.; Pop, M.; Salzberg, S.L. Ultrafast and memory-efficient alignment of short DNA sequences to the human genome. Genome Biol. 2009, 10, R25. [CrossRef] [PubMed] 